\title{
Minibars in the Centers of Normal Galaxies
}

\author{
A. V. Zasov and O. K. Sil'chenko \\ Sternberg Astronomical Institute, University av. 13, Moscow 119899, \\ Russia
}

\begin{abstract}
Analysis of ionized gas velocity fields in the circumnuclear regions of 13 normal spiral galaxies has shown that about half of them reveal a discrepancy between the kinematical axis at $R<2^{\prime \prime}$ and the photometric or kinematical axes of the outer regions. This result indicates a high frequency of occurrence of non-axisymmetric gravitational potentials in the inner few hundred parsecs of galaxies. The possible nature of these minibars is discussed.
\end{abstract}

Nuclear bars, observed in the very centers of some spiral galaxies, are poorly understood features of galactic structure. Most of them were found by visual inspection of galaxy images (see Buta \& Crocker 1993 and references therein). It is generally accepted that they are related to nuclear rings formed in barred galaxies at the inner Lindblad resonance (ILR), although according to Buta \& Crocker (1993), some of them were found in non-barred spirals. The typical length of known nuclear bars is $1-3 \mathrm{kpc}$ for $\mathrm{H}_{0}=75 \mathrm{~km} \mathrm{~s}^{-1} \mathrm{Mpc}^{-1}$, although their number (especially of the smallest ones) is certainly strongly affected by selection effects. Here we consider the presence of the smallest bar-like configurations in the centers of galaxies, which we call minibars.

Perhaps the most direct way to reveal a small bar-like pattern in the bright central region of a galaxy is to analyze velocity data to be sure that the rotation of gas (or stars) bears signs of a non-axisymmetric potential. Below we describe results of our measurements of ionized gas line-of-sight velocities in the inner $\left(R<10^{\prime \prime}\right)$ regions of nearby spiral galaxies obtained at the $6 \mathrm{~m}$ reflector of the Special Astrophysical Observatory of Russian Academy of Sciences mainly in 1993-1994.

Our observational program included 13 barred and non-barred nearby spiral galaxies without noticeable nuclear activity. All galaxies were chosen after preliminary analysis of our long-slit data obtained earlier which has revealed the presence of local features on their rotation curves in the circumnuclear regions such as local maxima, a steep gradient or a small plateau in the center. Three different spectral devices were used: a Long-Slit Spectrograph (LS), Multi-Pupil Field Spectrograph (MPFS) and Fabry-Perot scanning interferometer (FPI). Raw spectra were detected by a TV Photon Counting System $512 \times 512$ pixels $(1 \mathrm{px}=0.7-1.22)$. Typical seeing during the observations was $1.5-2^{\prime \prime}$.

For every galaxy we have used $\mathrm{H} \alpha$ and $[\mathrm{NII}]$ emission lines to measure central line-of sight velocity gradients along different position angles $(P A)$ in the range $R=1 . " 5-2 . " 3$ from the nucleus. The velocity field cannot be obtained by this way in detail, but this procedure makes it possible to verify its kinematical symmetry by comparing the position of its kinematical axis $P A_{o}$ with the kine- 
matical or photometric major axes found independently for the outer parts of a galaxy. For circularly rotating gas, a central line-of-sight velocity gradient alters with the $P A$ following a cosine-like law and reaches its extrema at the line of nodes. The presence of a bar or oval orbit distortion results in the shift of the extremum positions which we tried to find. The results are presented in Tables 1 and 2 .

Table 1. Program galaxies

\begin{tabular}{crrr}
\hline NGC & Type & Spectral device & $\begin{array}{r}\text { Presence } \\
\text { of minibar }\end{array}$ \\
\hline 23 & SBb & LS, MPFS, FPI & No \\
497 & SBbc & LS, MPFS & Yes \\
615 & $\mathrm{Sb}$ & LS, MPFS & No \\
895 & $\mathrm{Sc}$ & LS, MPFS & Yes \\
972 & $\mathrm{Sb}$ & LS, MPFS, FPI & Yes \\
4100 & $\mathrm{Sc}$ & LS & Yes \\
4536 & $\mathrm{SBc}$ & LS, MPFS & No \\
6181 & $\mathrm{SBc}$ & LS, MPFS, FPI & Yes \\
6643 & $\mathrm{Sc}$ & LS & No \\
7013 & $\mathrm{SO} / \mathrm{a}$ & LS & No \\
7171 & $\mathrm{SBb}$ & LS, MPFS & Yes (?) \\
7217 & $\mathrm{Sb}$ & MPFS & Yes \\
7331 & $\mathrm{Sbc}$ & LS & No \\
\hline \hline
\end{tabular}

A minibar is assumed to be detected if the discrepancy between kinematical major axis positions in the inner and in the outer regions exceeds $5-7^{\circ}$. As one can see from Table 1 , about half of the galaxies under consideration might have minibars. Only three of them possess large-scale bars, so we may guess that there is no direct relation between the two features. In general, although our results are not free from observational selection, they give first evidence of a high frequency of minibar occurrence among normal spiral galaxies.

Differences between the inner and outer kinematical major axes are presented in Table 2. Linear radii corresponding to the inner $P A_{o}$ are also given. The scales of the bar-like orbit distortions in these galaxies are among the smallest ever observed in galaxies beyond the Local Group (in most cases they are less than $0.5 \mathrm{kpc}$ ). Optical images of all these galaxies look quite normal in their inner parts. Two of them (NGC 4100 and NGC 7217) have nuclear rings less than $1 \mathrm{kpc}$ in radius (Buta \& Crocker 1993), but none has an optical nuclear bar mentioned in the literature (although detailed photometry of their nuclear regions is still highly desirable). In some cases (NGC 497, NGC 7171, NGC 7217) the difference of $P A_{o}$ 's is between $90^{\circ}$ and $270^{\circ}$ that mimics nuclear gas counterrotation under long-slit spectral observations.

Figure 1 presents some examples of azimuthal dependencies of line-of-sight velocity gradients in the nuclear regions of galaxies which are found to have minibars. Their maxima are noticeably shifted from the lines of nodes defined by the outer parts. In principle, the observed shift of cosine-like curve maxima 
Table 2. Galaxies with bar-like distortions of circumnuclear velocity ficlds

\begin{tabular}{crrrr}
\hline NGC & $\triangle P A_{\circ}$ & $R(\mathrm{kpc})$ & Comments & $\left(M_{b} / M_{d}\right)_{R=1 \mathrm{kpc}}$ \\
\hline 497 & $202^{\circ}$ & 1 & counterrotation? & 1.7 \\
895 & $44^{\circ}$ & 0.3 & inclined disk? & $>1$ \\
972 & $45^{\circ}$ & 0.2 & & 8 \\
& $10^{\circ}$ & 0.9 & & \\
4100 & $26^{\circ}$ & 0.15 & nuclear ring $R=6^{\prime \prime}$ & $>1$ \\
6181 & $28^{\circ}$ & 0.3 & & 1.4 \\
& $8^{\circ}$ & 1.0 & & \\
7171 & $190^{\circ}::$ & 0.35 & counterrotation? & 2.3 \\
7217 & $130^{\circ}$ & 0.15 & nuclear ring $R=10^{\prime \prime}$ & 2.0 \\
\hline
\end{tabular}
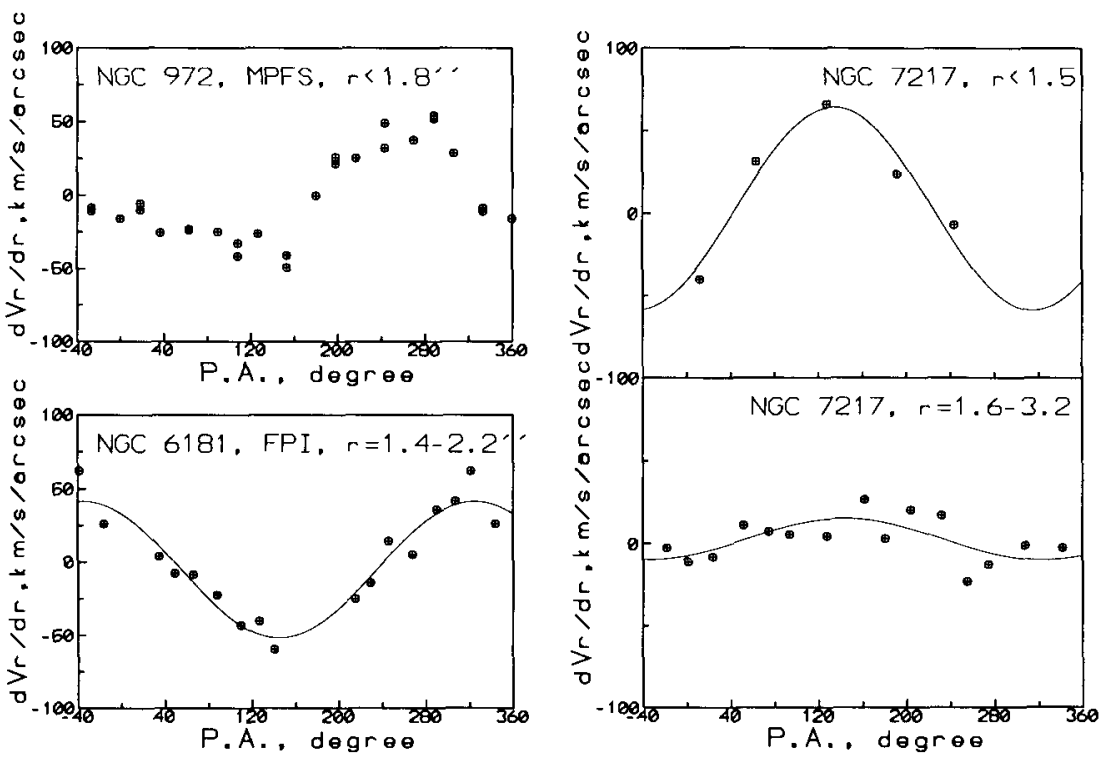

Figure 1. The azimuthal dependencies of central line-of-sight velocity gradients for three galaxies with minibars. The cosine curves are fitted by the least-square method. The lines of nodes defined by the outer disk isophotes are the following: for NGC $972 P A_{o}=152^{\circ}, 232^{\circ}$, for NGC $6181 P A_{o}=175^{\circ}, 355^{\circ}$, and for NGC $7217 P A_{o}=87^{\circ}, 267^{\circ}$. 

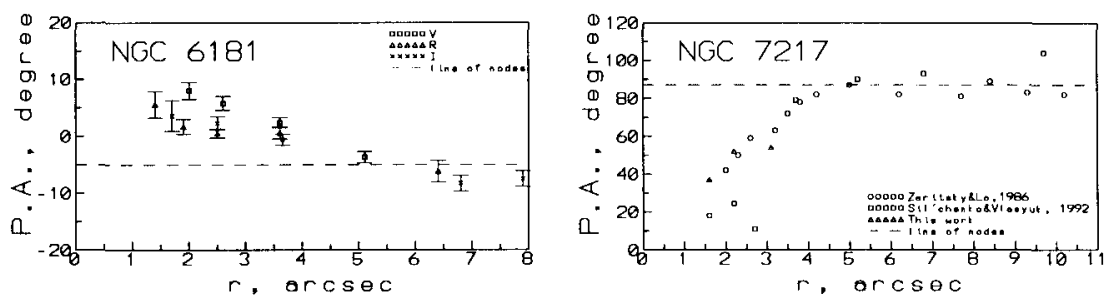

Figure 2. Photometric data: isophotal major axis twists along the radius. The dashed lines mark the orientations of the line of nodes at large radii.

may be caused not only by an oval orbit distortion, but also by the inclination of the inner disk with respect to the main disk of the galaxy. For several galaxies whose inner isophotes demonstrate orientations of the major axis in agreement with the kinematical central $P A_{o}$ (NGC 497, NGC 895, and NGC 972) this possibility cannot be excluded. However the azimuthal dependence of the central velocity gradient for NGC 972 (Figure 1) looks asymmetrical, so in this galaxy the presence of an inclined, circularly rotating nuclear disk is quite improbable. In addition there are other cases, e.g. NGC 6181 and 7217, where the inner isophotes are turned with respect to the line of nodes in the direction opposite to that of the kinematical major axis twist (the runs of photometric major axis along the radius obtained from our broad-band observations for NGC 6181 and from the combined data for NGC 7217 are shown in Figure 2). Here the presence of bar-like distortions is obvious. For two galaxies which were observed with FPI (NGC 972 and NGC 6181) we were able to trace the change of kinematical major axis orientation along the $R$ to obtain a boundary of the abnormal axis orientation region. In both cases it appears to be well resolved being about $5^{\prime \prime}-8^{\prime \prime}(0.6-0.9 \mathrm{kpc})$ for NGC 972 and about $5^{\prime \prime}(0.8 \mathrm{kpc})$ for NGC 6181 . Beyond these regions the influence of the minibar on the gaseous disk rotation is negligible.

The galaxies where minibars are found do not differ much from non-barred galaxies by the general shapes of their rotation curves. No color peculiarities of nuclear regions were also found for galaxies for which multiaperture $U B V$-data are available (Longo \& Vaucouleurs 1983, 1985).

One may expect that the main stellar disk does not control the gravitational potential of galaxies in their central regions. To verify it we modelled rotation curves of the galaxies within their growing parts (several kpc from the center) in the frame of a two-component model (exponential disk + sphere) to separate them (the extended rotation curve of NGC 7217 was taken from Rubin et al. 1985 , the others are from our observations). Photometric scale-lengths of the disks were taken from the literature, then we extrapolated the density distribution laws of the disks onto the central regions. The mass ratios of spherical and disk components for $R=1 \mathrm{kpc}$ are given in the last column of Table 2. It shows that in the central regions of the galaxies, gravitation of the stellar disk is suppressed by the bulge. This circumstance allows us to restrict the possible mechanisms of minibar formation. 
The large relative mass of the spherical component prevents the development of the classical bar-mode instability of the main disk. Two possibilities are left instead. The first one is the presence of dynamically decoupled massive nuclear disks in the inner several hundred parsecs, where a classical bar-mode may be developed. The idea of dense circumnuclear stellar disks is not in conflict with the observations of the inner gas kinematics in spiral galaxies (Afanasiev, Sil'chenko, \& Zasov 1989).

The second possibility is to consider the observed inner bars as slowly rotating bars developed from the hot stellar population through the elongated orbit instability (the mechanism proposed by Lynden-Bell 1979, and later developed by Polyachenko with coauthors - see Polyachenko 1994, Polyachenko \& Polyachenko 1994). In this case the process of sticking of elongated orbits together leads to the formation of a bar from the hot stellar population of the inner bulge or nuclear stellar disk. It is expected that a slow bar ends up at the ILR, so it may naturally explain the position of nuclear rings where they encircle nuclear bars. If a large-scale bar and a slow minibar coexist in a galaxy, there must not be any correlation between their orientations because of the different angular velocities, in accordance with observations of galaxies with nuclear bars.

In both cases the presence of large-scale "normal" bars is not necessary for minibars to be formed, although indirectly a large bar may provoke the creation of an inner one owing to the accumulation of bar-driven interstellar gas onto the central disk (if it exists) or to the dynamical heating of stars in the central region of the galaxy, which is essential for the formation of a slow bar.

Acknowledgments. We are grateful to our colleagues from the Special Astrophysical Observatory of Russian Academy of Sciences V. Afanasiev, A. Burenkov, S. Dodonov, and V. Vlasyuk for the invaluable help in carrying out the observational program.

This work has been partly supported by the Russian Fund of Fundamental Researches (grant 93-02-17060).

\section{References}

Afanasiev, V. L., Sil'chenko O. K., \& Zasov, A. V. 1989, A\&A, 213, L9

Buta, R. \& Crocker, D. A. 1993, AJ, 105, 1344

Longo, G. \& de Vaucouleurs, A. 1983, A General Catalogue of Photoelectric Magnitudes and Colors in the U, B, V System. Austin: Univ. Texas Press

Longo, G. \& de Vaucouleurs, A. 1985, Supplement to the General Catalogue of Photoelectric Magnitudes and Colors of Galaxies in the U, B, V System. Austin: Univ. Texas Press

Lynden-Bell, D. 1979, MNRAS, 187, 101

Polyachenko, V. L. 1994, in Physics of the Gaseous and Stellar Disks of the Galaxy, I. R. King, San Francisco: ASP, 103

Polyachenko, V. L. \& Polyachenko, E. V. 1994, Pis'ma v Astron. Zh., 20, 491

Rubin, V. C., Burstein, D., Ford, W. K., \& Thonnard, N. 1985, ApJ, 289, 81 